\title{
Combined oral cyclophosphamide and bevacizumab in heavily pre-treated ovarian cancer
}

José Miguel Jurado $•$ Alfonso Sánchez $\cdot$ Bella Pajares $\cdot$ Elisabeth Pérez $\cdot$

Lorenzo Alonso • Emilio Alba

Published online: 28 September 2008

Erratum to: Clin Transl Oncol

DOI 10.1007/s12094-008-0254-7

Unfortunately, José Miguel Jurado García was erroneously cited among the authors, instead of José Miguel Jurado.

The right authors' list should be: "José Miguel Jurado, Alfonso Sánchez, Bella Pajares, Elisabeth Pérez, Lorenzo Alonso, Emilio Alba"

The online version of the original article can be found under DOI 10.1007/s12094-008-0254-7

J.M. Jurado $(\varangle) \cdot$ A. Sánchez · B. Pajares · E. Pérez ·

L. Alonso · E. Alba

Medical Oncology Service

Hospital Universitario Virgen de la Victoria

C/ Campus Universitario de Teatinos, s/n

ES-29010 Málaga, Spain

e-mail: josemi88@ hotmail.com 